DOI: $10.2478 /$ aussoc-2018-0007

\title{
Deborah Lupton: Digital Health. Critical and Cross-Disciplinary Perspectives
}

\author{
London-New York: Routledge, 2018 \\ Ágnes SÁNTHA \\ Sapientia Hungarian University of Transylvania, Cluj-Napoca, Romania \\ santhaagnes@ms.sapientia.ro
}

Deborah Lupton's brand new volume, titled Digital Health. Critical and CrossDisciplinary Perspectives, is the second in the Critical Approaches to Health series of Routledge. The volume fills a gap in social sciences in particular but in the scholarly literature of medical and information technological sciences as well. To date, this is the first systematic analysis of the large field of digital health, its manifestations, and impact on social life.

Digital health encompasses a range of technologies serving the interest of patients, healthcare providers, and entrepreneurs. "The term 'digital health' refers to a wide range of technologies directed at delivering healthcare, providing information to lay people and helping them share their experiences of health and illness, training and educating healthcare professionals, helping people with chronic illnesses to engage in self-care and encouraging others to engage in activities to promote their health and wellbeing and avoid illness" (Lupton 2028: 1). The realm of health and medicine has become a digitized domain of everyday living and is often either welcome or condemned as a subversive transformation of healthcare in our times. This dichotomy of welcoming and condemning new technology is present from the very beginning till the very end of the analysis.

Besides acknowledging its huge potential, Lupton investigates the social, cultural, and political underpinnings of digital health. She writes a critical approach to digital health as she reveals how this is enmeshed within broader social relations and structures, showing more general considerations of power, interest, and benefit served by new technologies which function (also) to sustain structures of disadvantage and marginalization.

The author discusses several topics originating in this wide-ranging field. Three major aspects, however, are clearly marked out: first, the problems related to data protection, to the surveillance of individuals facing the invisible power; second, the persistent or even increasing social inequalities due to information 
technology in healthcare and disease prevention; and third the alleged transformation of medical professions as a result of the emerging and spreading digital technologies.

New technologies generate streams of digital data about human bodies. Online searching for health and medical information on the Internet is probably the simplest and most popular digital practice, gaining popularity in spite of the doubts on the accuracy of information. Telemedicine as a remote healthcare consultation is emerging as a time-, energy-, and cost-saving alternative to the classic doctor-patient setting. Digitized patient self-care and self-monitoring in chronic diseases, sensors, robotic care providers, and medical apps support patients in their daily self-care. Persuasive computing and gamification as ways of addiction preventions or motivators for lifestyle change also contribute to patient empowerment. This kind of empowerment and cost efficiency are seen as positive outcomes of digital health technologies. Through this constant dataveillance of the body, the concept of the "digitally engaged patient" entails a shift of responsibility from care providers to individuals.

Precisely within the context of the new EU data protection regulations, big data and cloud computing systems raise questions about data privacy and storage. Digital media technologies have expanded the active creation and sharing of content, including that of personal information; and this carries considerable risks. Data has been largely commoditized, measuring and quantifying has become the supreme value, more powerful than other means of collecting information, often neglecting individual needs and qualitative aspects. Here, Lupton takes up the concept of surveillance. For Foucault, power is not only repressive but has a productive nature, his terms biopolitics and biopower suggesting practices of managing, regulating, and monitoring human bodies. Instead of coercion and violence to ensure discipline, there are more subtle forms of power that encourage citizens to conform to expectations and norms. Digital surveillance in our days is similar to the Foucaultian concept of biopolitics, it often entails self-surveillance as a technology of the self, which, in turn, monitors human bodies from the power side. This raises concerns about privacy, information ethics, and the misuse of personal data as digital health data are primary resources for commercialization and commodification through big enterprises, with the aim of developing new products and services.

Dataveillance strategies reinforce existing social inequalities. Geographical location, state of health, and socioeconomic status influence digital technology use. Within Europe, in some countries, searching for medical information on the Internet is a widely used activity, while in others only a minority is benefiting from it. The contrasts between the most and the least developed regions in the world are even more striking. People with disabilities are sometimes, indeed, helped and empowered in their communication by the new digital technologies 
(Caron \& Light 2015, Dobransky \& Hargittai 2016), but to the most part the disabled are rather locked out from their use. Gender differences in digital device usage start in early childhood. Women are disadvantaged in the male-dominated world of information technology, the more so in developing countries. Girls and women are also more exposed to cyberbullying than men, and other social minorities also suffer several forms of online harassment. Self-tracking devices can be empowering in the case of the elderly, helping them achieve mobility at old age; however, they might substitute human care and interaction and, as such, sustain their isolation.

Datasets used by data brokers generate information on the customers' lifestyle choices, which result in chances for some and exclusion for others, specifically for those who are disadvantaged in the first place. Besides the privacy concerns raised by too much online visibility, invisibility is also dangerous. It exacerbates the disadvantages of invisible social groups in the process of decision making based on digital data, their needs remain unidentified, and thus they are left out of eventual resource allocation (Lerman 2013).

One of the most valuable chapters of the book is the last one, discussing the aspects of digital technologies in medical professions, most notably the reluctance of health professionals to the expansion of digital health devices. Digital health was and still is perceived as a threat from the health practitioners' side as it undermines the high prestige of medical profession and the asymmetry of the doctor-patient relationship.

Thanks to new technologies, health surveillance practices have evolved considerably and communication with patients and colleagues have become easier for doctors. However, the professionals' resistance is obvious for at least four reasons introduced by the author. First, digital technologies starting with the simplest one, online information searching, are being perceived as a challenge to the prestige and autonomy of medical professions, being incompatible with the existing paternalistic, pedagogical, and often repressive orientation towards patients. Second, medical profession loses control over medical knowledge itself, the profession being alarmed at the often misused lay medical treatments online. Third, doctors are often being defamed by online comments to the medical service patients receive, which are the hardest to refute. Fourth, the online public sphere offers the possibility for public debate such as for the public expression of opposing views to the public health policy decisions.

From the patients' view, telemedical technologies are welcome because they are convenient, cost-saving, reduce waiting hours, the time and effort spent on patient face-to-face consultation, yet the quality of care is perceived as lower compared to the classic healthcare setting (Andreassen et al. 2006). With all its possibilities, complete trust in these services has not yet been achieved. The example shows that in spite of the troublesome new phenomena disquieting 
doctors and challenging their power, medical profession still seems to hold its position. Patients who search for medical information online clearly claim that they need doctors' real-time advice even on Internet sites and express their need for the customization of information available online. This supports the argument that biomedicalization, medical knowledge and authority - even if challenged by new digital technologies - remain dominant within the new setting. No need for doctors to worry about losing their prestige.

It is not enough to read Deborah Luptons' book only once. The author reports on every mentionable study in the field, mostly from the Western world and Australia, her home country, without neglecting, however, the specific issue of digital health in developing countries. Thanks to its huge empirical material, the series of examples, and evidence standing behind every thesis she formulates, this book needs to be invoked over and over again.

\section{References}

Andreassen, Hege K., Tronsden, Marianne, Kummervold, Per Egil, Gammon, Deede et al. 2006. Patients Who Use E-Mediated Communication with Their Doctor: New Constructions of Trust in the Patient-Doctor Relationship. Qualitative Health Research 16(2): 238-248.

Caron, Jessica, Light, Janice. 2015. "My World Has Expanded Even Though I'm Stuck at Home": Experiences of Individuals with Amyotrophic Lateral Sclerosis Who Use Augmentative and Alternative Communication and Social Media. American Journal of Speech-Language Pathology 24(4): 680-695.

Dobransky, Kerry, Hargittai, Eszter. 2016. Unrealized Potential: Exploring the Digital Disability Divide. Poetics 58: 18-28.

Lerman, Jonas. 2013. Big Data and Its Exclusions. Stanford Law Review Online (available at: https://www.stanfordlawreview.org/online/privacy-and-bigdata-big-data-and-its-exclusions). 